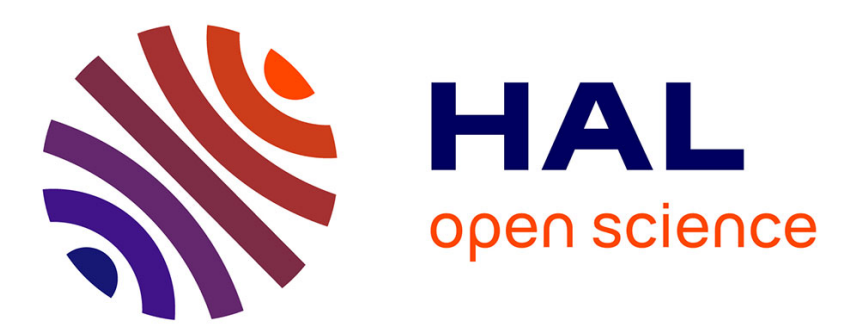

\title{
Complete Lattice Structure of Poincaré Upper-Half Plane and Mathematical Morphology for Hyperbolic-Valued Images
}

Jesus Angulo, Santiago Velasco-Forero

\section{- To cite this version:}

Jesus Angulo, Santiago Velasco-Forero. Complete Lattice Structure of Poincaré Upper-Half Plane and Mathematical Morphology for Hyperbolic-Valued Images. First International Conference on Geometric Science of Information (GSI'2013), Aug 2013, Paris, France. pp.535 - 542, 10.1007/978-3-64240020-9_59 . hal-01536381

HAL Id: hal-01536381

https://hal-mines-paristech.archives-ouvertes.fr/hal-01536381

Submitted on 11 Jun 2017

HAL is a multi-disciplinary open access archive for the deposit and dissemination of scientific research documents, whether they are published or not. The documents may come from teaching and research institutions in France or abroad, or from public or private research centers.
L'archive ouverte pluridisciplinaire HAL, est destinée au dépôt et à la diffusion de documents scientifiques de niveau recherche, publiés ou non, émanant des établissements d'enseignement et de recherche français ou étrangers, des laboratoires publics ou privés. 


\title{
Complete lattice structure of Poincaré upper-half plane and mathematical morphology for hyperbolic-valued images
}

\author{
Jesús Angulo $^{1}$ and Santiago Velasco-Forero ${ }^{2}$ \\ 1 CMM-Centre de Morphologie Mathématique, \\ Mathématiques et Systèmes, MINES ParisTech, France \\ 2 ITWM - Fraunhofer Institute, Kaiserlautern, Germany \\ jesus.angulo@mines-paristech.fr; velascoforero@itwm.fraunhofer.de
}

\begin{abstract}
Mathematical morphology is a nonlinear image processing methodology based on the application of complete lattice theory to spatial structures. Let us consider an image model where at each pixel is given a univariate Gaussian distribution. This model is interesting to represent for each pixel the measured mean intensity as well as the variance (or uncertainty) for such measurement. The aim of this paper is to formulate morphological operators for these images by embedding Gaussian distribution pixel values on the Poincaré upper-half plane. More precisely, it is explored how to endow this classical hyperbolic space with partial orderings which lead to a complete lattice structure.
\end{abstract}

\section{Introduction}

This work is motivated by the exploration of a mathematical image model $f$ where instead of having a scalar intensity $t \in \mathbb{R}$ at each pixel $p$, i.e., $f(p)=t$, we have a univariate Gaussian probability distribution of intensities $N\left(\mu, \sigma^{2}\right) \in \mathcal{N}$, i.e., image $f$ is defined as the function

$$
f:\left\{\begin{array}{l}
\Omega \rightarrow \mathcal{N} \\
p \mapsto N\left(\mu, \sigma^{2}\right)
\end{array}\right.
$$

where $\Omega$ is the support space of pixels $p$ (e.g., for $2 \mathrm{D}$ images $\Omega \subset \mathbb{Z}^{2}$ ) and $\mathcal{N}$ denotes the family of univariate Gaussian probability distribution functions (pdf). Henceforth, the corresponding image processing operators should be able to deal with Gaussian distributions-valued pixels. In particular, morphological operators for images $f \in \mathcal{F}(\Omega, \mathcal{N})$ involves that the space of Gaussian distributions $\mathcal{N}$ must be endowed of a partial ordering leading to a complete lattice structure. In practice, it means that given a set of Gaussian pdfs, we need to be able to define a Gaussian pdf which corresponds to the infimum (inf) of the set and another one to the supremum (sup). Mathematical morphology is a nonlinear image processing methodology based on the computation of sup/inf-convolution filters (i.e., dilation/erosion operators) in local neighborhoods [12]. Mathematical morphology is theoretically formulated in the framework of complete lattices and operators defined on them $[11,9]$. 
We are inspired here by an information geometry viewpoint [3], which is based on considering that the univariate Gaussian pdfs are points in a hyperbolic space [6,10]. For a deep flavor on hyperbolic geometry see [5]. There are several models representing the hyperbolic space.There exists an isometric mapping between any pair among these models and analytical transformations to convert from one to other are well known $[5,10]$. In this paper, we focus on the simplest Poincaré half-plane model, $\mathcal{H}^{2}$, which is sufficient for our practical purposes of manipulating Gaussian pdfs. In summary, from a theoretical viewpoint, the aim of this paper is to endow $\mathcal{H}^{2}$ with partial orderings which lead to useful invariance properties in order to formulate appropriate morphological operators for images $f: \Omega \rightarrow \mathcal{H}^{2}$. This paper is a summary of a more developed study available in [1].

\section{Geometry of Poincaré upper-half plane $\mathcal{H}^{2}$}

In complex analysis, the upper-half plane is the set of complex numbers with positive imaginary part: $\mathcal{H}^{2}=\{z=x+i y \in \mathbb{C} \mid y>0\}$. We use also the notation $x=\Re(z)$ and $y=\Im(z)$. The boundary of upper-half plane is the real axis together with the infinity.

Riemannian metric and distance. In hyperbolic geometry, the Poincaré upper-half plane model (originated with Beltrami and also known as Lobachevskii space in Soviet scientific literature) is the space $\mathcal{H}^{2}$ together with the Poincaré metric $\left(g_{k l}\right)=\operatorname{diag}\left(\frac{1}{y^{2}} \frac{1}{y^{2}}\right)$ such that the hyperbolic arc length is given by $d s^{2}=$ $\frac{d x^{2}+d y^{2}}{y^{2}}=\frac{|d z|^{2}}{y^{2}}=y^{-1} d z y^{-1} d z^{*}$.

The distance between two points $z_{1}=x_{1}+i y_{1}$ and $z_{2}=x_{2}+i y_{2}$ in $\left(\mathcal{H}^{2}, d s^{2}\right)$ is the function

$$
\operatorname{dist}_{\mathcal{H}^{2}}\left(z_{1}, z_{2}\right)=\cosh ^{-1}\left(1+\frac{\left(x_{1}-x_{2}\right)^{2}+\left(y_{1}-y_{2}\right)^{2}}{2 y_{1} y_{2}}\right)
$$

Distance (1) is derived from the logarithm of the cross-ratio between these two points and the points at the infinity. From the latter formulation is easy to check that for two points with $x_{1}=x_{2}$ the distance is $\operatorname{dist}_{\mathcal{H}^{2}}\left(z_{1}, z_{2}\right)=\left|\log \left(\frac{y_{1}}{y_{2}}\right)\right|$.

Geodesics. The geodesics of $\mathcal{H}^{2}$ are the vertical lines, $V L(a)=\left\{z \in \mathcal{H}^{2}\right.$ $\Re(z)=a\}$, and the semi-circles in $\mathcal{H}^{2}$ which meet the horizontal axis $\Re(z)=0$ orthogonally, $S C_{r}(a)=\left\{z \in \mathcal{H}^{2}|| z-z^{\prime} \mid=r ; \Re\left(z^{\prime}\right)=a\right.$ and $\left.\Im\left(z^{\prime}\right)=0\right\}$. In particular, given any pair $z_{1}, z_{2} \in \mathcal{H}^{2}$, there is a unique geodesic connecting them, or in other terms, given these two points with $x_{1} \neq x_{2}$ there exists a unique semi-circle of center $c=(a, 0)$, radius $r$, and being orthogonal to x-axis, i.e., $\left(z_{1}, z_{2}\right) \mapsto S C_{r_{1 \frown 2}}\left(a_{1 \frown 2}\right)$ where

$a_{1 \frown 2}=\frac{x_{2}^{2}-x_{1}^{2}+y_{2}^{2}-y_{1}^{2}}{2\left(x_{2}-x_{1}\right)} ; r_{1 \frown 2}=\sqrt{\left(x_{1}-a_{1 \frown 2}\right)^{2}+y_{1}^{2}}=\sqrt{\left(x_{2}-a_{1 \frown 2}\right)^{2}+y_{2}^{2}}$. 
More precisely, the unique geodesic parameterized by the length, $t \mapsto \gamma\left(z_{1}, z_{2} ; t\right)$, $\gamma:[0,1] \rightarrow \mathcal{H}^{2}$ joining two points $z_{1}=x_{1}+i y_{1}$ and $z_{2}=x_{2}+i y_{2}$ such as $\gamma\left(z_{1}, z_{2} ; 0\right)=z_{1}$ and $\gamma\left(z_{1}, z_{2} ; 1\right)=z_{2}$ is given by

$$
\gamma\left(z_{1}, z_{2} ; 0\right)= \begin{cases}x_{1}+i e^{\xi t+t_{0}} & \text { if } x_{1}=x_{2} \\ {\left[r \tanh \left(\xi t+t_{0}\right)+a\right]+i\left[\frac{r}{\cosh \left(\xi t+t_{0}\right)}\right]} & \text { if } x_{1} \neq x_{2}\end{cases}
$$

with $a$ and $r$ given in (2) and where for $x_{1}=x_{2}, t_{0}=\log \left(y_{1}\right), \xi=\log \frac{y_{2}}{y_{1}}$ and for $x_{1} \neq x_{2}: t_{0}=\cosh ^{-1}\left(\frac{r}{y_{1}}\right)=\sinh ^{-1}\left(\frac{x_{1}-a}{y_{1}}\right), \xi=\log \left(\frac{y_{1}}{y_{2}} \frac{r+\sqrt{r^{2}-y_{2}^{2}}}{r+\sqrt{r^{2}-y_{1}^{2}}}\right)$.

Hyperbolic polar coordinates. The position of point $z=x+i y$ in $\mathcal{H}^{2}$ can be given either in terms of Cartesian coordinates $(x, y)$ or by means of polar hyperbolic coordinates $(\eta, \phi)$, where $\eta$ represents the distance of the point from the origin $\mathcal{O}_{\mathcal{H}^{2}}=(0,1)$ and $\phi$ represents the slope of the tangent in $\mathcal{O}_{\mathcal{H}^{2}}$ to the geodesic (i.e., semi-circle) joining the point $(x, y)$ with the origin. The formulas which relate the hyperbolic coordinates $(\eta, \phi)$ to the Cartesian ones $(x, y)$ are [4]

$$
\left\{\begin{array} { l } 
{ x = \frac { \operatorname { s i n h } \eta \operatorname { c o s } \phi } { \operatorname { c o s h } \eta - \operatorname { s i n h } \eta \operatorname { s i n } \phi } , \eta > 0 } \\
{ y = \frac { 1 } { \operatorname { c o s h } \eta - \operatorname { s i n h } \eta \operatorname { s i n } \phi } , - \frac { \pi } { 2 } < \phi < \frac { \pi } { 2 } }
\end{array} \quad \left\{\begin{array}{l}
\eta=\operatorname{dist}_{\mathcal{H}^{2}}\left(\mathcal{O}_{\mathcal{H}^{2}}, z\right) \\
\phi=\arctan \frac{x^{2}+y^{2}-1}{2 x}
\end{array}\right.\right.
$$

We notice that the center of the geodesic passing trough $(x, y)$ from $\mathcal{O}_{\mathcal{H}^{2}}$ has Cartesian coordinates given by $(\tan \phi, 0)$.

\section{Endowing $\mathcal{H}^{2}$ with partial ordering and its complete lattice structure}

The notion of ordering invariance in the Poincare upper-half plane was considered in the Soviet literature $[7,8]$. Ordering invariance with respect to simple transitive subgroup $T$ of the group of motions was studied, i.e., group $T$ consists of transformations $t$ of the form: $z=x+i y \mapsto z^{\prime}=(\lambda x+\alpha)+i \lambda y$, where $\lambda>0$ and $\alpha$ are real numbers. We named $T$ the Guts group. We introduce here partial orders in $\mathcal{H}^{2}$ and study invariance properties to transformations of Guts group or to subgroups of $\mathrm{SL}(2, \mathbb{R})$ (Möbius transformations).

Upper half-plane polar ordering. Let us introduce a total ordering in $\mathcal{H}$ based on hyperbolic polar coordinates, which takes into account an ordering relationship with respect to $\mathcal{O}_{\mathcal{H}^{2}}$. Given a pair of points $\forall z_{1}, z_{2} \in \mathcal{H}$, the upper half-plane polar ordering states

$$
z_{1} \leq_{\mathcal{H}^{2}}^{\text {pol }} z_{2} \Leftrightarrow\left\{\begin{array}{l}
\eta_{1}<\eta_{2} \\
\eta_{1}=\eta_{2}
\end{array} \text { or } \text { and } \tan \phi_{1} \leq \tan \phi_{2}\right.
$$

The polar infimum $\left(z_{1} \wedge_{\mathcal{H}^{2}}^{\text {pol }} z_{2}\right)$ and supremum $\left(z_{1} \vee_{\mathcal{H}^{2}}^{\text {pol }} z_{2}\right)$ are naturally defined from the order (5); and are naturally extended for any subset of points $Z=$ 
$\left\{z_{k}\right\}_{1 \leq k \leq K}$, denoted by $\bigwedge_{\mathcal{H}^{2}}^{\text {pol } Z}$ and $\bigvee_{\mathcal{H}^{2}}^{\text {pol }} Z$. Total order $\leq_{\mathcal{H}^{2}}^{\text {pol }}$ leads to a complete lattice, bounded from the bottom (i.e., the origin $\mathcal{O}_{\mathcal{H}^{2}}$ ) but not from the top. Furthermore, as $\leq_{\mathcal{H}^{2}}^{\text {pol }}$ is a total ordering, the supremum and the infimum will be either $z_{1}$ or $z_{2}$.

Polar total order is invariant to any Möbius transformation $M_{g}$ which preserves the distance to the origin (isometry group) and more generally to isotone maps in distance, i.e., $\eta\left(z_{1}\right) \leq \eta\left(z_{2}\right) \Leftrightarrow \eta\left(M_{g}\left(z_{1}\right)\right) \leq \eta\left(M_{g}\left(z_{2}\right)\right)$ but which also preserves the orientation order, i.e., order on the polar angle. This is for instance the case of orientation group $\mathrm{SO}(2)$ and the scaling maps $z \mapsto M_{g}(z)=\lambda z$, $0<\lambda \in \mathbb{R}$.

Upper half-plane geodesic ordering. As discussed above, there is a unique hyperbolic geodesic joining any pair of points. Given two points $z_{1}, z_{2} \in$ $\mathcal{H}^{2}$ such that $x_{1} \neq x_{2}$, let $S C_{r_{1-2}}\left(a_{1-2}\right)$ be the semi-circle defining their geodesic, where the center $a_{1 \frown 2}$ and the radius $r_{1 \frown 2}$ are given by Eqs. (4). Let denote by $z_{1 \frown 2}$ the point of $S C_{r_{1-2}}\left(a_{1 \frown 2}\right)$ having maximal imaginary part, i.e., its imaginary part is equal to the radius: $z_{1 \frown 2}=a_{1 \frown 2}+i r_{1 \frown 2}$.

The upper half-plane geodesic ordering $\preceq_{\mathcal{H}^{2}}^{\text {geo }}$ defines an order for points being in the same half of their geodesic semi-circle as follows,

$$
z_{1} \preceq_{\mathcal{H}^{2}}^{\text {geo }} z_{2} \Leftrightarrow\left\{\begin{array}{l}
a_{1 \frown 2} \leq x_{1}<x_{2} \text { or } \\
x_{2}<x_{1} \leq a_{1 \frown 2}
\end{array}\right.
$$

Property of transitivity of this partial ordering, i.e., $z_{1} \preceq_{\mathcal{H}^{2}}^{\text {geo }} z_{2}, z_{2} \preceq_{\mathcal{H}^{2}}^{\text {geo }} z_{3} \rightarrow$ $z_{1} \preceq_{\mathcal{H}^{2}}^{\text {geo }} z_{3}$, holds for points belonging to the same geodesic. For two points in a geodesic vertical line, $x_{1}=x_{2}$, we have $z_{1} \preceq_{\mathcal{H}^{2}}^{\text {geo }} z_{2} \Leftrightarrow y_{2} \leq y_{1}$. According to this partial ordering, we define the geodesic infimum, denoted by $\curlywedge_{\mathcal{H}^{2}}^{\text {geo }}$, as the point on the geodesic joining $z_{1}$ and $z_{2}$ with maximal imaginary part, i.e., for any $z_{1}, z_{2} \in \mathcal{H}^{2}$, with $x_{1} \neq x_{2}$, we have

$$
z_{1} \curlywedge_{\mathcal{H}^{2}}^{\text {geo }} z_{2} \Leftrightarrow\left\{\begin{array}{cl}
\left(x_{1} \vee x_{2}\right)+i\left(y_{1} \vee y_{2}\right) & \text { if } x_{1}, x_{2} \leq a_{1 \frown 2} \\
\left(x_{1} \wedge x_{2}\right)+i\left(y_{1} \vee y_{2}\right) & \text { if } x_{1}, x_{2} \geq a_{1} \frown 2 \\
z_{1 \frown 2} & \text { otherwise }
\end{array}\right.
$$

If $x_{1}=x_{2}$, we have that $z_{1} \curlywedge_{\mathcal{H}^{2}}^{\text {geo }} z_{2}=x_{1}+i\left(y_{1} \vee y_{2}\right)$. In any case, we have that $\operatorname{dist}_{\mathcal{H}^{2}}\left(z_{1}, z_{2}\right)=\operatorname{dist}_{\mathcal{H}^{2}}\left(z_{1}, z_{1} \curlywedge_{\mathcal{H}^{2}}^{\text {geo }} z_{2}\right)+\operatorname{dist}_{\mathcal{H}^{2}}\left(z_{1} \curlywedge_{\mathcal{H}^{2}}^{\text {geo }} z_{2}, z_{2}\right)$. Intuitively, we notice that the geodesic infimum is the point of the geodesic farthest from the real line.

We observe that if one attempts to define the geodesic supremum from the partial ordering $\preceq_{\mathcal{H}^{2}}^{g e o}$, it results that the supremum is not defined for any pair of points, i.e., supremum between $z_{1}$ and $z_{2}$ is defined only if and only if both points are in the same half of its semi-circle. To tackle this limitation, we propose to define the geodesic supremum $z_{1} \curlyvee_{\mathcal{H}^{2}}^{\text {geo }} z_{2}$ by duality with respect to the following involution in $\mathcal{H}^{2}$ :

$$
z \mapsto \complement z=-x+i y^{-1} .
$$

Hence, we have the geodesic supremum given by

$$
z_{1} \curlyvee_{\mathcal{H}^{2}}^{\text {geo }} z_{2}=\complement\left(\complement z_{1} \curlywedge_{\mathcal{H}^{2}}^{\text {geo }} \complement z_{2}\right)
$$




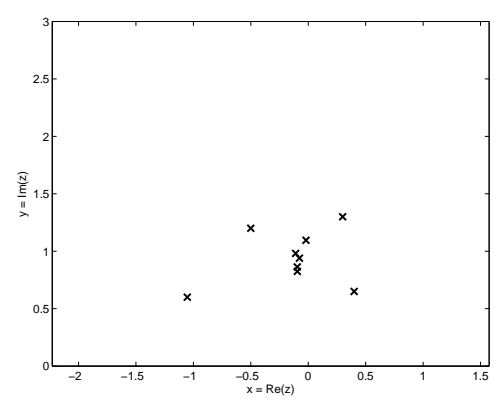

(a)

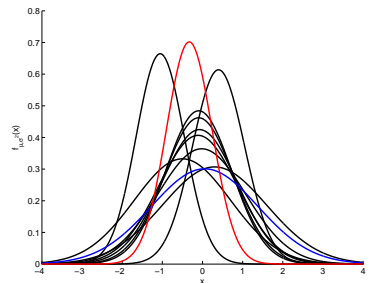

(c)

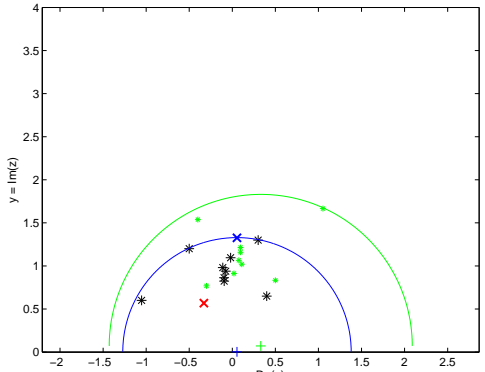

(b)

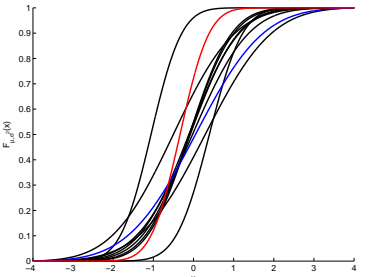

(d)

Fig. 1. (a) Set of nine points in $\mathcal{H}^{2}, Z=\left\{z_{k}\right\}_{1 \leq k \leq 9}$. (b) Computation of infimum $\bigwedge_{\mathcal{H}^{2}}^{\text {geo }} Z=z_{\text {inf }}$ (blue " $\times$ ") and supremum $\bigvee_{\mathcal{H}^{2}}^{\text {geo }} Z=z_{\text {sup }}$ (red " $\times$ "). Black "*" are the original points and green " $*$ " the corresponding dual ones. (c) In black, set of Gaussian pdfs associated to $Z$, i.e., $N_{k}\left(\mu=x_{k}, \sigma^{2}=y_{k}^{2}\right)$; in green, infimum Gaussian pdf $N_{\text {inf }}\left(\mu=x_{\text {inf }}, \sigma^{2}=y_{\text {inf }}^{2}\right)$; in red, supremum Gaussian pdf $N_{\text {inf }}\left(\mu=x_{\text {inf }}, \sigma^{2}=y_{\text {inf }}^{2}\right) .($ d $)$ Cumulative distribution functions of Gaussian pdfs from (c).

Nevertheless, in order to have a structure of complete lattice for $\left(\mathcal{H}^{2}, \preceq_{\mathcal{H}^{2}}^{\text {geo }}\right)$, it is required that the infimum and the supremum of any set of points $Z=$ $\left\{z_{k}\right\}_{1 \leq k \leq K}$ with $K>2$, are well defined. Namely, according to (7), the geodesic infimum of $Z$, denoted $\bigwedge_{\mathcal{H}^{2}}^{g e o} Z$, corresponds to the point $z_{\text {inf }}$ with maximal imaginary part on all possible geodesics joining any pair of points $z_{n}, z_{m} \in Z$. In geometric terms, that means that between all these geodesics, there exists one which gives $z_{\text {inf }}$. Instead of computing all the geodesics, we propose to define the infimum $\bigwedge_{\mathcal{H}^{2}}^{g e o} Z$ as the point $z_{\text {inf }}=a_{\text {inf }}+i r_{\text {inf }}$, where $a_{\text {inf }}$ is the center of the smallest semi-circle in $\mathcal{H}^{2}$ of radius $r_{\text {inf }}$ which encloses all the points in the set $Z$. We have the following property $\bigwedge_{\mathcal{H}^{2}}^{g e o} Z=z_{\text {inf }} \preceq_{\mathcal{H}^{2}}^{g e o} z_{k}, 1 \leq k \leq K$, which geometrically means that the geodesic connecting $z_{\text {inf }}$ to any point $z_{k}$ of $Z$ lies always in one of the half part of the semi-circle defined by $z_{\text {inf }}$ and $z_{k}$.

In practice, the minimal enclosing semi-circle defining $z_{\text {inf }}$ can be easily computed by means of the following algorithm based on the minimum enclosing Euclidean circle $M E C$ of a set of points: (1) Working on $\mathbb{R}^{2}$, define a set of points given, on the one hand, by $Z$ and, on the other hand, by $Z^{*}$ which corresponds to the reflected points with respect to $x$-axis (complex conjugate), i.e., points $Z=\left(x_{k}, y_{k}\right)$ and points $Z^{*}=\left(x_{k},-y_{k}\right), 1 \leq k \leq K$; (2) Compute the 
$\operatorname{MEC}\left(Z \cup Z^{*}\right) \mapsto C_{r}(c)$, in such a way that, by symmetric point configuration, we necessarily have the center on the $x$-axis, i.e., $c=\left(x_{c}, 0\right)$; (3) The infimum $\bigwedge_{\mathcal{H}^{2}}^{g e o} Z=z_{\text {inf }}$ is given by $z_{\text {inf }}=x_{c}+i r$. Fig. 1(a)-(b) gives an example of computation of the geodesic infimum from a set of points in $\mathcal{H}^{2}$.

As for the case of two points, the geodesic supremum of $Z$ is defined by duality with respect to involution (8), i.e.,

$$
z_{\text {sup }}=\bigvee_{\mathcal{H}^{2}}^{\text {geo }} Z=\complement\left(\bigwedge_{\mathcal{H}^{2}}^{\text {geo }} \complement Z\right)=a_{\text {sup }}+i r_{\text {sup }},
$$

with $a_{\text {sup }}=-x_{c}^{\text {dual }}$ and $r_{\text {sup }}=1 / r^{\text {dual }}$, where $S C_{r_{\text {dual }}}\left(x_{c}^{\text {dual }}\right)$ is the minimal enclosing semi-circle from dual set of points $\complement Z$. According to this formulation by duality we have that, for any $Z \subset \mathcal{H}^{2}, z_{\text {inf }} \preceq_{\mathcal{H}^{2}}^{\text {geo }} z_{\text {sup }}$, which is a consequence of the fact $z_{\text {sup }}$ lies inside the semi-circle defined by $z_{\text {inf }}$. An example of computing the geodesic supremum $z_{\text {sup }}$ is also given in Fig. 1(a)-(b).

It is easy to see that geodesic infimum and supremum have the following properties: (i) $\Im\left(z_{\text {inf }}\right) \geq \Im\left(z_{k}\right)$ and $\Im\left(z_{\text {sup }}\right) \leq \Im\left(z_{k}\right), \forall z_{k} \in Z$; (ii) $\bigvee_{1 \leq k \leq K} \Re\left(z_{k}\right)<$ $\Re\left(z_{\text {inf }}\right), \Re\left(z_{\text {sup }}\right)<\bigwedge_{1<k<K} \Re\left(z_{k}\right)$. The proofs are straightforward from the notion of minimal enclosing semi-circle. An interpretation of the geodesic infimum and supremum for a set of Gaussian pdfs is also given in Fig. 1.

Geodesic infimum and supremum being defined by minimal enclosing semicircles, their invariance properties are related to homothetic transformations as well as translation on $x$-axis. That corresponds just to the Guts group of transformations.

Upper half-plane asymmetric geodesic infimum/supremum. According to the properties of geodesic infimum $z_{\text {inf }}$ and supremum $z_{\text {sup }}$ discussed above, we note that their real parts $\Re\left(z_{\text {inf }}\right)$ and $\Re\left(z_{\text {sup }}\right)$ belong to the interval bounded by the real parts of points of set $Z$. Moreover, $\Re\left(z_{\text {inf }}\right)$ and $\Re\left(z_{\text {sup }}\right)$ are not ordered between them. Therefore, the real part of supremum can be smaller than that of the infimum. For instance, in the extreme case $Z=\left\{z_{k}\right\}_{1 \leq k \leq K}$, if $y_{k}=y, 1 \leq k \leq K$, we have $\Re\left(z_{\text {inf }}\right)=\Re\left(z_{\text {sup }}\right)=1 / K \sum_{k=1}^{K} x_{k}$. From the viewpoint of morphological image filtering, it can be potentially interesting to impose an asymmetric behavior for the infimum and supremum such that $\Re\left(z_{\text {inf }}^{-\overrightarrow{+}}\right) \leq z_{k} \leq \Re\left(z_{\text {sup }}^{-\overrightarrow{+}}\right), 1 \leq k \leq K$. Note that the proposed notation $-\rightarrow+$ indicates a partially ordered set on x-axis. In order to fulfil these requirements, we can geometrically consider the rectangle bounding the minimal enclosing semi-circle, which is just of dimensions $2 r_{\text {inf }} \times r_{\text {inf }}$, and use it to define the asymmetric infimum $z_{\mathrm{inf}}^{-\overrightarrow{+}}$ as the upper-left corner of the rectangle. The asymmetric supremum $z_{\text {sup }}^{-\rightarrow+}$ is similarly defined from the bounding rectangle of the dual minimal enclosing semi-circle. Mathematically, we have:

$$
\left\{\begin{array}{c}
z_{\mathrm{inf}}^{-\rightarrow}=\bigvee_{\mathcal{H}^{2}}^{-\rightarrow+} Z=\left(a_{\mathrm{inf}}-r_{\mathrm{inf}}\right)+i r_{\mathrm{inf}} \\
z_{\mathrm{sup}}^{-\rightarrow+}=\bigwedge_{\mathcal{H}^{2}}^{-\rightarrow} Z=-\left(x_{c}^{\text {dual }}-r^{\text {dual }}\right)+i \frac{1}{r^{\text {dual }}}
\end{array}\right.
$$




\section{Morphological operators on $\mathcal{F}\left(\Omega, \mathcal{H}^{2}\right)$ for processing univariate Gaussian distribution-valued images}

If $\left(\mathcal{H}^{2}, \leq\right)$ is a complete lattice, the set of images $\mathcal{F}\left(\Omega, \mathcal{H}^{2}\right)$ is also a complete lattice defined as follows: for all $f, g \in \mathcal{F}\left(\Omega, \mathcal{H}^{2}\right)$, (i) $f \leq g \Leftrightarrow f(p) \leq g(p)$, $\forall p \in \Omega$; (ii) $(f \wedge g)(p)=f(p) \wedge g(p), \forall p \in \Omega$; (iii) $(f \vee g)(p)=f(p) \vee g(p)$, $\forall p \in \Omega$, where $\wedge$ and $\vee$ are the infimum and supremum in $\mathcal{H}^{2}$. One can now define the following adjoint pair of flat erosion $\varepsilon_{B}(f)$ and flat dilation $\delta_{B}(f)$ of each pixel $p$ of the image $f[11,9]$ :

$$
\varepsilon_{B}(f)(p)=\bigwedge_{q \in B(p)} f(p+q), \text { and } \delta_{B}(f)(p)=\bigvee_{q \in B(p)} f(p-q)
$$

where the set $B$ is called the structuring element, which defines the set of points in $\Omega$ when it is centered at point $p$, denoted $B(p)$ [12]. These operators, which are translation invariant, can be seen as constant-weight (this the reason why they are called flat) inf/sup-convolutions, where the structuring element $B$ works as a moving window. The above erosion (resp. dilation) moves object edges within image in such a way that expands image structures with values in $\mathcal{H}^{2}$ close to the bottom element (resp. close to the top) of the lattice $\mathcal{F}\left(\Omega, \mathcal{H}^{2}\right)$ and shrinks object with values close to the top element (resp. close to the bottom).

Given the adjoint image operators $\left(\varepsilon_{B}, \delta_{B}\right)$, the opening and closing by adjunction of image $f$, according to structuring element $B$, are defined as the product operators $[11,9]$ :

$$
\gamma_{B}(f)=\delta_{B}\left(\varepsilon_{B}(f)\right) \text {, and } \varphi_{B}(f)=\varepsilon_{B}\left(\delta_{B}(f)\right) \text {. }
$$

Openings and closings are referred to as morphological filters, which remove objects of image $f$ that do not comply with a criterion related, on the one hand, to the invariance of the object support to the structuring element $B$ and, on the other hand, to the values of the object on $\mathcal{H}^{2}$ which are far from (in the case of the opening) or near to (in the case of the closing) to the bottom element of $\mathcal{H}^{2}$ according to the given partial ordering $\leq$. Once the pairs of dual operators $\left(\varepsilon_{B}, \delta_{B}\right)$ and $\left(\gamma_{B}, \varphi_{B}\right)$ are defined, the other morphological filters and transformation can be naturally defined [12] for images in $\mathcal{F}\left(\Omega, \mathcal{H}^{2}\right)$. We limit here the the illustrative examples with the basic ones.

Example. Fig. 2 illustrates an example of image enhancement from a very noisy image $g(p)$. The noise is related to an acquisition at the limit of exposure time/spatial resolution. We consider an image model $f(p)=f_{x}(p)+i f_{y}(p)$, where $f_{x}(p)=g(x)$ and $f_{y}(p)$ is the standard deviation of intensities in a patch of radius equal to 4 pixels centered a $p$. Results obtained from a closing $\varphi_{B}(f)(p)$ using the polar ordering-based lattice, the geodesic lattice $\left(\mathcal{H}^{2}, \curlyvee_{\mathcal{H}^{2}}^{\text {geo }}\right)$ and the asymmetric geodesic lattice $\left(\mathcal{H}^{2}, \bigwedge_{\mathcal{H}^{2}}^{-\rightarrow}, \bigvee_{\mathcal{H}^{2}}^{-+}\right)$are compared, where the structuring element $B$ is a square of $5 \times 5$ pixels. In order to be able to compare them with a non morphological operator, it is also given the result of filtering by computing the minimax center [2] in $\mathcal{H}^{2}$ in a square of $5 \times 5$ pixels. 


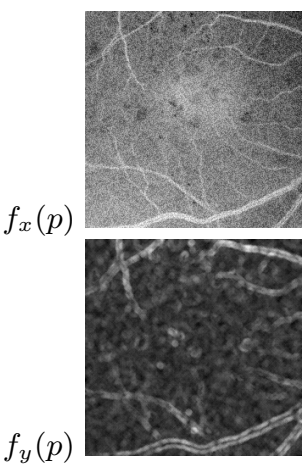

(a) $f(p)$
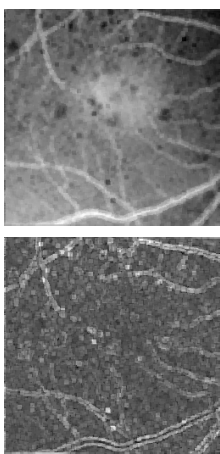

(b) Minimax center $5 \times 5$
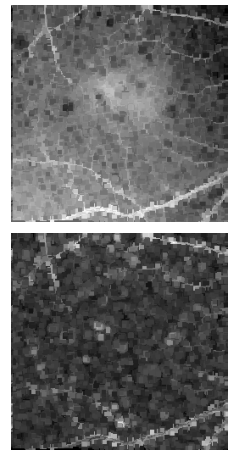

(c) $\varphi_{B}(f)(p)$, $\left(\mathcal{H}^{2}, \leq_{\mathcal{H}^{2}}^{\text {pol }}\right)$
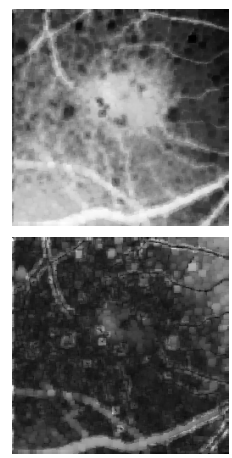

(d) $\varphi_{B}(f)(p)$, $\left(\mathcal{H}^{2}, \preceq_{\mathcal{H}^{2}}^{\text {geo }}\right)$
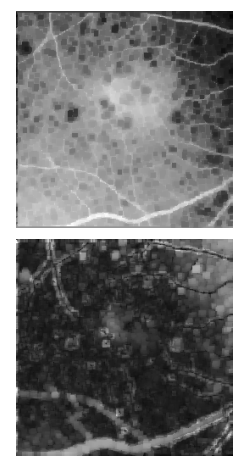

(e) $\varphi_{B}(f)(p)$, $\left(\mathcal{H}^{2}, \bigwedge_{\mathcal{H}^{2}}^{-{ }^{+}}, \bigvee_{\mathcal{H}^{2}}^{-\vec{p}^{+}}\right)$

Fig. 2. Morphological processing of Gaussian distribution-valued noisy image: (a) Original image $f \in \mathcal{F}\left(\Omega, \mathcal{H}^{2}\right)$, showing both the real (top row) and the imaginary (bottom row) components; (b) filtered image by computing the minimax center in a square of $5 \times 5$ pixels; (c) morphological closing working on the polar ordering-based lattice; (d) morphological closing working on the geodesic lattice; (e) morphological closing on the asymmetric geodesic framework. In the three cases the structuring element $B$ is also a square of $5 \times 5$ pixels.

\section{References}

1. J. Angulo, S. Velasco-Forero. Morphological processing of univariate Gaussian distribution-valued images based on Poincaré upper-half plane representation. hal00795012, v1, 25 p. 2013.

2. M. Arnaudon, F. Nielsen. On approximating the Riemannian 1-center. Computational Geometry, Vol. 46, No. 1, 93-104, 2013.

3. S. Amari, H. Nagaoka. Methods of Information Geometry, Translations of Mathematical Monographs. Vol. 191, Am. Math. Soc., 2000.

4. V. Cammarota, E. Orsingher. Travelling Randomly on the Poincaré Half-Plane with a Pythagorean Compass. Journal of Statistical Physics, 130(3): 455-482, 2008.

5. J.W. Cannon, W.J. Floyd, R. Kenyon, W.R. Parry. Hyperbolic Geometry. Flavors of Geometry, MSRI Publications, Vol. 31, 1997.

6. S.I.R. Costa, S.A. Santos, J.E. Strapasson. Fisher information distance: a geometrical reading, arXiv:1210:2354v1, 15 p., 2012.

7. A.K. Guts. Mappings of families of oricycles in Lobachevsky space. Math. USSRSb., Vol. 19, 131-138, 1973.

8. A.K. Guts. Mappings of an ordered Lobachevsky space. Siberian Math. J., Vol. 27, No. 3, 347-361, 1986.

9. H.J.A.M. Heijmans. Morphological image operators. Academic Press, Boston, 1994.

10. F. Nielsen, R. Nock. Hyperbolic Voronoi diagrams made easy. In Proc. of the 2010 IEEE ICCSA, pp. 74-80, 2010.

11. J. Serra. Image Analysis and Mathematical Morphology. Vol II: Theoretical Advances, Academic Press, London, 1988.

12. P. Soille. Morphological Image Analysis. Springer-Verlag, Berlin, 1999. 